\title{
Influence of Oxidative and/or Reductive Treatment on Human Hair (II): Effect of Hydrophilic Extracts from Rosmarinus officinalis L. on Oxidative and/or Reductive Hair-Damage
}

\author{
Koji TAKada $^{1}$, Keita Someya ${ }^{1 *}$, Naoko Matsuo ${ }^{1}$, \\ Yuichi NishIDA ${ }^{2}$ and Tatsuo HAYASHI ${ }^{1}$ \\ ${ }^{1}$ Biological Science Research Center, Lion Corporation \\ (Tajima 100, Odawara, Kanagawa 256-0811, JAPAN) \\ ${ }^{2}$ Beauty-Care Research Laboratories, Lion Corporation \\ (7-13-12 Hirai, Edogawa-ku, Tokyo 132-0035, JAPAN)
}

Edited by T. Itoh, Kitasato Univ., and accepted May 27, 2003 (received for review March 5, 2003)

\begin{abstract}
To confirm the effects of hydrophilic extracts from Rosmarinus officinalis L. (HER) on oxidative and/or reductive hair-damage, we first estimated the HER antioxidant effect by doing a $\beta$-carotene decoloring test. We additionally conducted an analysis of the biochemical parameters, for example cysteic acid or chemiluminescence (CL), and observed the morphological changes (snapping and splitting frequency) after chemical and physical hair treatment. Furthermore, we compared the effect of applying HER with the effect of applying no antioxidant or the effect of a typical hydrophilic antioxidant, ascorbic acid (AsA). HER had a strong antioxidant effect in the hydrophilic radical generation system in the $\beta$-carotene decoloring test. However, HER exhibited moderate antioxidant effects in the lipophilic radical generation system. Concerning hair-damage, applying HER suppressed cysteic acid in the hair after chemical and physical treatment more than when no antioxidant is applied. In addition, HER and AsA application also suppressed CL intensity in the damaged hair in the system using 2-methyl-6-(4-methoxyphenyl)-3,7-dihydroimidazo[1,2-a]pyrazin-3-one hydrochloride (MCLA). However, HER only exhibited a tendency for CL reduction in damaged hair when we induced $\mathrm{CL}$ with short time irradiation of ultraviolet rays (UV) without chemical reagents. Furthermore, HER and AsA suppressed the snapping and splitting frequency in the hair, and the suppression level of HER tended to be stronger than that of AsA. HER demonstrated the antioxidant effect in a hydrophilic radical generation system. Furthermore, HER could prevent oxidation and morphological changes in damaged hair that was subjected to oxidative and/or reductive treatment, such as perming and bleaching, as well as physical treatment, which is postulated to cause hair-damage in daily life.
\end{abstract}

Key words: hair-damage, Rosmarinus officinalis L., cysteic acid, chemiluminescence, snapping and splitting frequency

\footnotetext{
${ }^{*}$ Correspondence to: Keita SomEYA, Biological Science Research Center, Lion Corporation, Tajima 100, Odawara, Kanagawa 256-0811, JAPAN

E-mail: keisliof@lion.co.jp
} 


\section{Introduction}

We have previously shown that human hair is not only damaged by chemical stress (e.g. perming, dyeing, and bleaching) but also by physical or environmental stress in daily life (e.g. brushing, and sunlight-exposure) (1). We confirmed that a combination of both kinds of stress causes severe damage to hair and that oxidative and reductive stress are significant factors regarding hair-damage.

It is well known that oxidative stress causes lipid peroxidation and this peroxidation has received a great deal of attention in connection with its pathological effects and its role in aging and in diseases such as atherosclerosis and cancer (2-10). Our previous study (1) revealed that oxidative stress was related not only to inner biological tissues but also to outer organs, such as human hair, and we suggested that antioxidants could be effective in treating and preventing hair-damage.

Rosmarinus officinalis L. is a labiate plant about $1 \mathrm{~m}$ high and grows on the Mediterranean Sea coast. Leaves of this plant have been used as a cooking herb and the essential oil has been used as an ingredient in perfumes. Furthermore, it has been reported that this plant shows antimicrobial activity and a superior antioxidant effect $(11,12)$. Several active ingredients have been isolated and identified for their antioxidant effect $(13,14)$. The lipophilic component has been found to be carnosol and the hydrophilic component is rosmarinic acid.

In this study, we estimated the effect of the hydrophilic extracts from Rosmarinus officinalis L. (HER) on oxidative and reductive hair-damage by analyzing the biochemical parameters (e.g. cysteic acid, and chemiluminescence). Chemiluminescence (CL) detection is particularly and specifically sensitive to peroxide and reflects the oxidative level in biological tissue clearly $(15,16)$. We recently succeeded in analyzing CL in human hair and we were able to evaluate the antioxidant effect of HER on hair-damage. We also estimated the effect of HER on the morphological changes in damaged hair, such as the frequency of snapping and splitting.

\section{Experimental}

\section{$\mathbf{2} \cdot 1$ Preparation of HER}

HER was prepared from Rosmarinus officinalis L. in the following manner. Leaves from the plant were infused in ten times their volume of $80 \%$ ethanol. This infusion was then heated at $50^{\circ} \mathrm{C}$ for $3 \mathrm{~h}$. The extract was concentrated to one-thirtieth of its original volume using a rotary evaporator. Water was added to the concentration and thoroughly mixed. The volume of water was ten times that of the concentration. The mixture was then left at room temperature for $1 \mathrm{~h}$. The supernatant was separated from the mixture by filtration. This supernatant was evaporated in vacuo until dry (using a rotary evaporator) and was used as the HER dried sample. The sample contained hydrophilic antioxidants, with about $10 \%$ rosmarinic acid content.

\subsection{Chemicals and Hair Sample}

Linoleic acid and 2-methyl-6-(4-methoxyphenyl)3,7-dihydroimidazo[1,2-a]pyrazin-3-one hydrochloride (MCLA) were purchased from Sigma Chemical Co. (St. Louis, MO, USA). Tocopherol (Toc), which mainly contains the $\gamma$-and $\delta$ - types, were purchased from Nisshin Oil Mills Ltd. (Tokyo, Japan). Other chemicals were purchased from Wako Pure Chemical Industries (Osaka, Japan). The hair sample was the same as described in our previous paper (1).

\section{$2 \cdot 3 \quad \beta$-Carotene Decoloring Test}

We conducted a $\beta$-carotene decoloring test as outlined by Someya et al. (17). We dissolved $0.5 \mathrm{mg}$ of $\beta$ carotene, $20 \mathrm{mg}$ of linoleic acid, and $200 \mathrm{mg}$ of Tween \#40 in $2 \mathrm{~mL}$ of chloroform in a lipophilic radical generating system. Chloroform was removed with a nitrogen gas stream. One hundred $\mathrm{mL}$ of $20 \mathrm{mM}$ phosphate buffer ( $\mathrm{pH}$ 6.8) was added to the dried mixture and was shaken to prepare the micelle solution. Fifty $\mu \mathrm{L}$ of the HER solution was added to $5 \mathrm{~mL}$ of the micelle solution and incubated at $50^{\circ} \mathrm{C}$. We measured the absorbance at $470 \mathrm{~nm}$ at constant intervals and calculated the half life period of the $\beta$-carotene. The antioxidant effect of the samples was evaluated by comparing the half life period with the control period.

The micelle solution was prepared again but without the linoleic acid in the hydrophilic radical generating system. The sample solution and $5 \mathrm{mM}$ of 2,2' -azobis(2-amidinopropane hydrochloride) (AAPH), which was the hydrophilic radical generator, were added to the micelle solution and incubated at $37^{\circ} \mathrm{C}$. The half life period of the $\beta$-carotene was measured and the antioxidant effect of the samples was evaluated using the 
same method as before.

\subsection{Chemical Treatment, Hair Brushing and Application of Antioxidant Samples}

Chemical treatment and brushing were conducted as detailed in our previous paper (1), with brushing carried out 5000 times. HER or ascorbic acid (AsA) was dissolved in $20 \%$ ethanol with $0.25 \%$ concentration. The hair tress was soaked in the antioxidant solution for $30 \mathrm{~s}$ immediately or $7 \mathrm{~d}$ after chemical treatment, and then dried at $40^{\circ} \mathrm{C}$ for $15 \mathrm{~min}$.

\subsection{Cysteic Acid Analysis}

We conducted cysteic acid analysis of the hair samples as detailed in our previous paper (1).

\subsection{Analysis Using MCLA}

We conducted a CL analysis using MCLA, as outlined by Kigawa et al. (18), with some slight modifications. The condition of the hair tresses used is described in section $\mathbf{2} \cdot \mathbf{4}$. The hair tress was cut from the tip end, where the length was $2 \mathrm{~cm}$ and the weight $0.04 \mathrm{~g}$. This cut hair was soaked in $2 \mathrm{~mL}$ of $1 \%$ Tween \#40 for $1 \mathrm{~h}$. MCLA was then added until the final concentration was $100 \mathrm{nM}$, and the CL was measured for 5 min using an AQUACOSMOS/VIM system (Hamamatsu Photonics K.K., Hamamatsu, Japan). The CL intensity for $5 \mathrm{~min}$ was integrated and calculated to be the total photon count, and the integrated luminescence image was analyzed.

\section{$2 \cdot 7$ Analysis of CL Induced by UV Irradiation}

We conducted the CL measurement without chemical enhancers using a chemiluminescence analyzer CLA210 (Tohoku Electrical Industrial Co., Ltd., Sendai, Japan). The hair tress sample was put in a $60 \mathrm{~mm}$ diameter stainless steel petri dish. Ultraviolet rays (UV) were exposed to the hair tress at a $5 \mathrm{~cm}$ distance for $5 \mathrm{~s}$ using a UV lamp, a BLAK-RAY MODEL UVL56 (Ultra Violet products, Inc., San Gabriel, CA, USA). The CL measurement was started $5 \mathrm{~s}$ after exposure to eliminate the influence of chemiluminescence from the singlet oxygen, and the half life period of the intensity was calculated. The half life period of the CL intensity of no hair sample were also measured in the same manner. We defined the difference in the half life period between the hair sample and the no hair sample as the oxidation value of the hair sample. A typical CL chart is shown in Fig. 1.

\section{$2 \cdot 8$ Measurement of Frequency of Snap- ping and Splitting}

After the chemical treatment and sample application that were described in section $\mathbf{2} \cdot \mathbf{4}$, the hair tress was brushed 5000 times, and morphological changes were observed using a microscope. The numbers of snapped

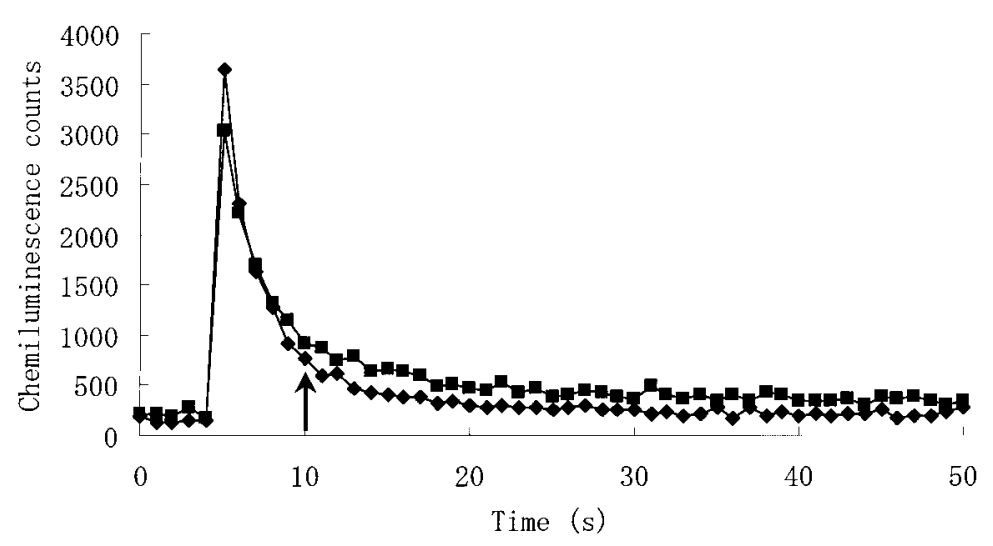

Fig. 1 CL Chart for Untreated or Chemically Treated Hair. Chemical treatment was performed by perming and two application of bleaches (PBB), as described in the experimental section. The hair tress was exposed for $5 \mathrm{~s}$ with a handy UV lamp, and $5 \mathrm{~s}$ after exposure the CL measurement was started. $\diamond$, Untreated hair; $\mathbf{\square}$, chemically treated hair; $\uparrow$, start point of measurement. 
and split ends were counted and their frequencies were calculated.

\section{$2 \cdot 9$ Statistical Analysis}

Any significant differences in the mean values between groups were determined by using an analysis of variance (ANOVA) and Fisher's protected least significant difference (PLSD).

\section{Results}

\section{$3 \cdot 1$ Antioxidant Effect of HER in the $\beta$ - carotene Decoloring Test}

Figure 2 shows the half life period for the $\beta$-carotene decoloring test where lipophilic radicals were generated. The concentration in each sample was $1 \mathrm{ppm}$. Toc $(p<0.01)$ had a significantly longer half life period compared to the control. In this experiment, HER had a weak effect and AsA had no effect.

Figure 3 shows the half life period for the $\beta$-carotene decoloring test where hydrophilic radicals were generated. The concentration in each sample was $1 \mathrm{ppm}$. HER $(p<0.01)$ and AsA $(p<0.01)$ had a significantly longer half life period than the control. In this experiment, the antioxidant effect for Toc and AsA were the

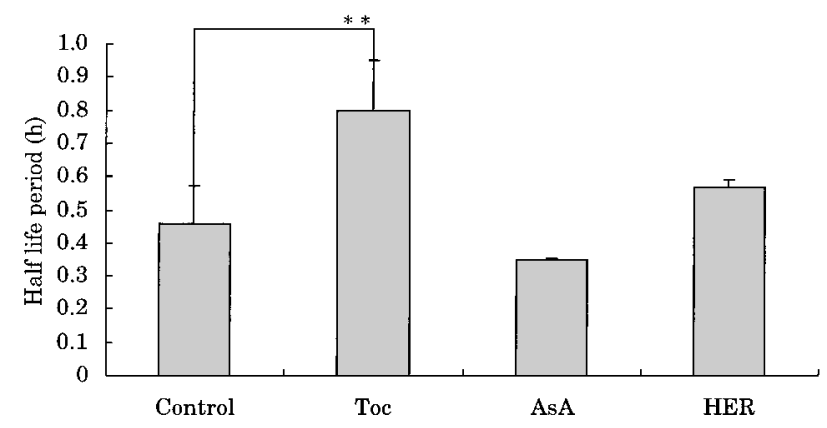

Fig. 2 Antioxidant Effects in $\beta$-Carotene Decoloring Test where Oil-Soluble Radicals were Generated. $\beta$ Carotene was dispersed in the micelle system using Tween \#40 and each sample (at a concentration of 1 ppm) was added to it. No sample was added to the control. The decoloration of $\beta$-carotene in the micelle was induced by oil-soluble radicals that were generated by the autoxidation of the linoleic acid. $\beta$-Carotene was measured by absorbance at $470 \mathrm{~nm}$ and the half life period of the $\beta$-carotene was calculated. Data was taken from the $\mathrm{M} \pm \mathrm{SD}$ of 3 samples. ** represents significantly different $(p<0.01)$ from the control. reverse of that experienced with the lipophilic radical generating system. HER had the strongest effect, AsA was the next strongest, and Toc was the weakest.

\subsection{Cysteic Acid in Hair after Chemical and Physical Treatments}

Table 1 shows the cysteic acid in the hair with or without HER application after chemical and physical treatment. HER application immediately after chemical treatment $(p<0.05)$ exhibited significantly less systeic acid in the hair than that in hair with no HER application. HER application $7 \mathrm{~d}$ after chemical treatment exhibited a tendency to decrease systeic acid in hair compared to that in no HER application. The value, however, was not significant.

\subsection{Using MCLA after Treatment}

The luminescence image of the hair was observed using MCLA, which was able to enhance the chemiluminescence. All hair samples were treated chemically and physically as described in the experimental section without untreated hair. Permed and twice bleached hair (PBB) exhibited a greater amount of apparent luminescence compared to untreated hair (UT). On the other hand, HER application hair suppressed the CL and this intensity was similar to that of UT (Fig. 4).

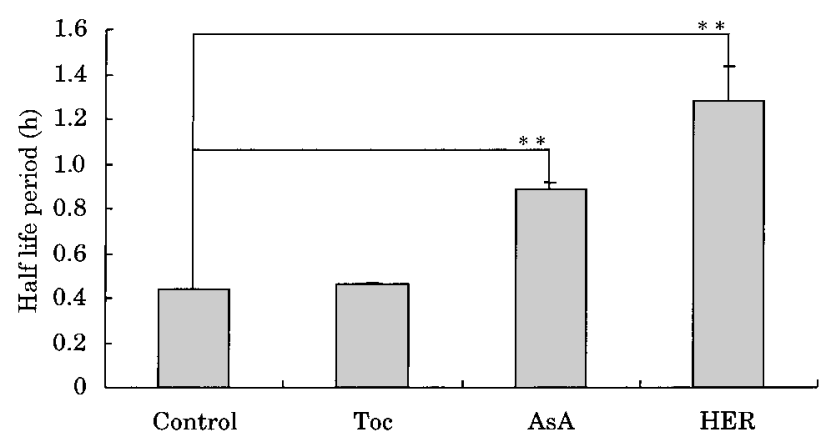

Fig. 3 Antioxidant Effects in the $\beta$-Carotene Decoloring Test where Water-Soluble Radicals were Generated. $\beta$-Carotene was dispersed in the micelle system using Tween \#40 and each sample (at a concentration of $1 \mathrm{ppm}$ ) was added to it. No sample was added to the control. The decoloration of $\beta$ carotene in the micelle was induced by watersoluble AAPH radicals. $\beta$-Carotene was measured by absorbance at $470 \mathrm{~nm}$ and the half life period of $\beta$-carotene was calculated. Data was taken from the $\mathrm{M} \pm \mathrm{SD}$ of 3 samples. ** represents significantly different $(p<0.01)$ from the control. 
Table 1 Degree of Cysteic Acid in Hair after Chemical and Physical Treatment.

\begin{tabular}{lcc}
\hline \multicolumn{1}{c}{ Samples } & $\mathrm{n}^{1}$ & $\begin{array}{c}\text { Degree of cysteic acid } \\
(\mathrm{mol} \%)^{2}\end{array}$ \\
\hline Chemical treatment & 6 & $4.52 \pm 0.42$ \\
+HER immediately after the treatment & 6 & $4.02 \pm 0.18^{3}$ \\
+HER seven days after the treatment & 6 & $4.27 \pm 0.20$ \\
\hline
\end{tabular}

Chemical treatment was carried out as described in the experimental section. The HER solution $(0.25 \%$ concentration $)$ was applied to the hair sample immediately and also 7 days after the treatment. All samples were brushed as described in the experimental section seven days after the chemical treatment. The cysteic acid was then measured as described in the experimental section. ${ }^{1}$ represents the number of analyzed samples. ${ }^{2} \mathrm{M} \pm \mathrm{SD} .{ }^{3}$ represents a significant difference $(p<0.05)$ to the without HER application group.

The CL counts were integrated and these counts in the hair samples were compared in the 5 min measurement. The results shown in Fig. 5 reflect the luminescent images that were obtained in Fig. 4. PBB $(p<0.01)$ exhibited a significantly higher level of CL than UT. On the other hand, HER $(p<0.01)$ and AsA $(p<0.01)$ significantly suppressed the CL compared to PBB.

\subsection{Analysis by UV Irradiation}

Furthermore, we attempted to analyze CL in hair without a chemical reagent, which enhances CL intensity. We conducted the CL analysis in hair that was subjected to UV irradiation, and we also measured the oxi- dation level of the hair using this analysis system. The $\mathrm{CL}$ peak that originated from the singlet oxygen was first detected. This peak decayed gradually. However, the decaying curves were different for untreated hair and chemically treated hair (Fig. 1). Therefore, we evaluated the oxidation level of hair and the effect of HER.

As shown in Fig. 6, PBB exhibited higher oxidation values than UT $(p<0.01)$, and HER application tended to exhibit a suppression of the values. However, AsA application did not exhibit apparent suppression.

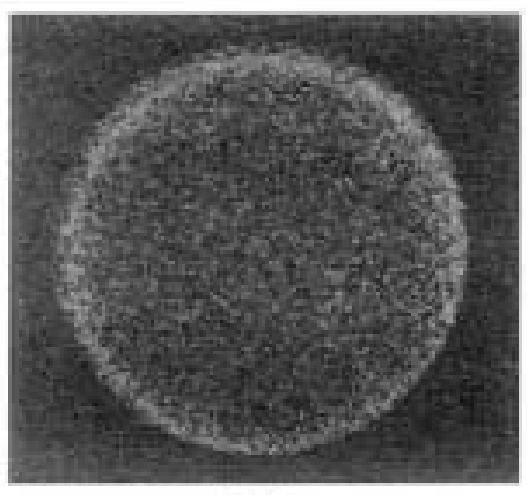

UT

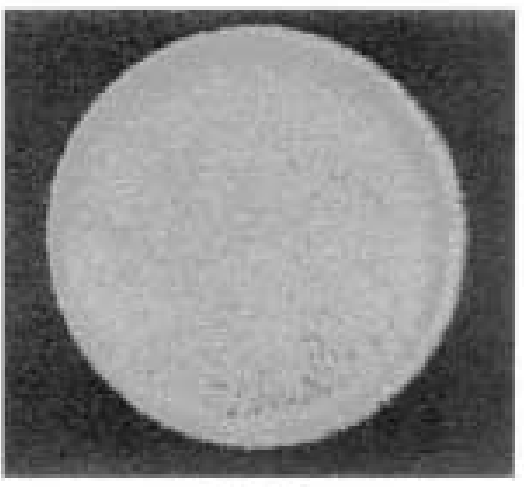

PBB

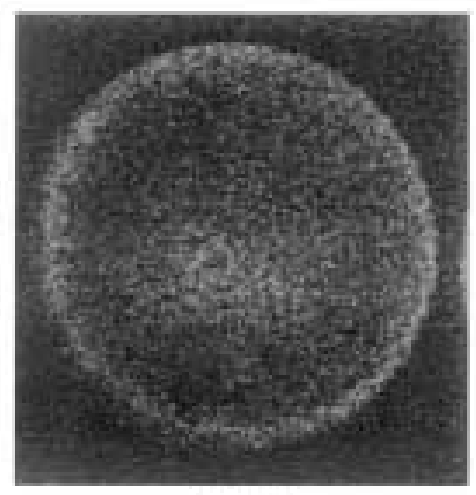

+ HER

Fig. 4 Luminescence Image of Hair Samples Using MCLA. Chemical treatment and brushing were carried out as described in the experimental section. HER solution $(0.25 \%$ concentration) was applied to the hair sample immediately after the chemical treatment. MCLA (100 nM concentration) was added to each hair sample 1 $\mathrm{h}$ after soaking in $1 \%$ Tween $\# 40$ and CL from these samples was analyzed. UT, Untreated hair; PBB, perming and two application of bleaches; +HER, HER application after PBB. 


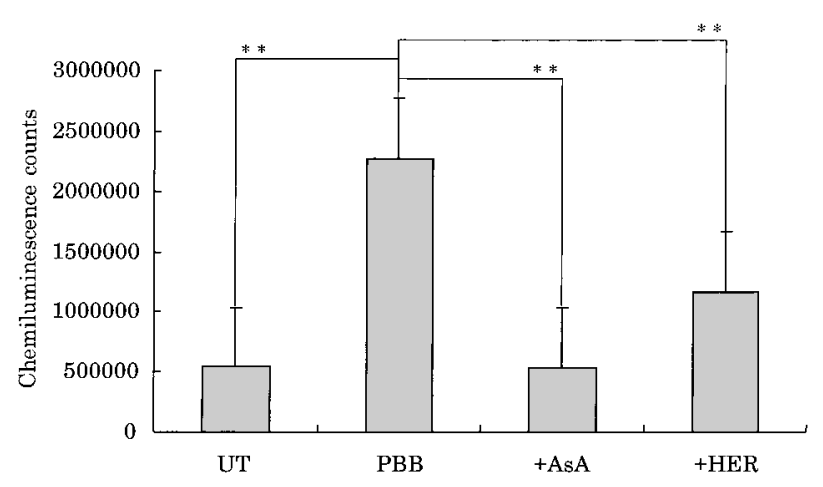

Fig. 5 Antioxidant Effect of HER as Estimated by CL Analysis Using MCLA. The analysis approach and the concentration of antioxidant are the same as described for Fig. 4. All CL counts were integrated for the 5 min measurement. +AsA, AsA application after PBB. Other abbreviations are the same as described in Fig. 4. Data was taken from the $\mathrm{M} \pm$ $\mathrm{SD}$ of 3 samples. ** represents significantly different $(p<0.01)$ to PBB.

\subsection{Frequency of Snapping and Splitting Ends after Treatment}

We also conducted morphological observation, which was the splitting and snapping frequency in the hair tress after the chemical and physical treatment. The physical treatment, the brushing, was carried out seven days after the chemical treatment and the antioxidant application, as described in the experimental section.

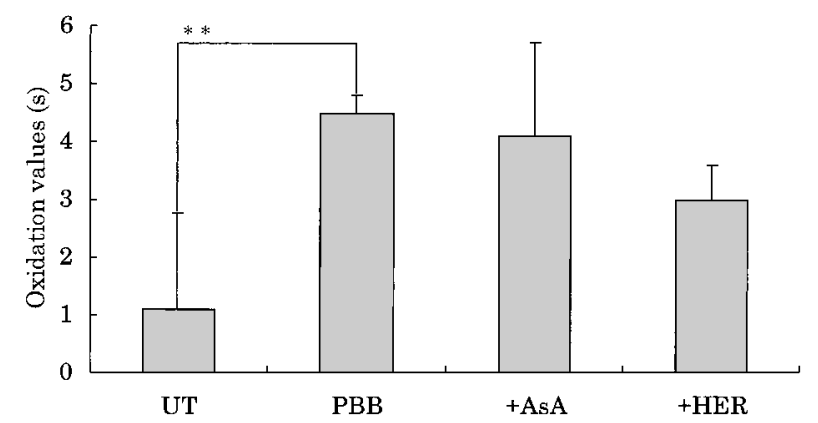

Fig. 6 Antioxidant Effects in CL Analysis where Induced by UV Irradiation. The analysis was performed in the same way as Fig. 1 and the concentration of antioxidant was the same as Fig. 4. The oxidation value was calculated as described in the experimental section. Abbreviations are the same in Fig. 4 and 5. Data was taken from the $M \pm S D$ of 4 samples. $* *$ represents significantly different $(p<0.01)$ from PBB.
As shown in Fig. 7, HER $(p<0.01)$ and AsA $(p<0.01)$ significantly suppressed the snapping and splitting frequency in the hair compared to PBB after the brushing. HER exhibited a higher suppression of the snapping and splitting frequency than AsA.

\section{Discussion}

We demonstrated that HER, which had an antioxidant effect in a hydrophilic radical generation system, could prevent oxidation and morphological changes in damaged hair that was subjected to oxidative and/or reductive treatment.

The $\beta$-carotene decoloring test was conducted with a heterogeneous emulsion system. The oxidative stability of lipids and the strength of antioxidants were quite different to those under homogeneous conditions (19-24). HER had a strong antioxidant effect in the $\beta$-carotene emulsion system, which generated hydrophilic radicals (Fig. 3). On the other hand, HER did not exhibit such a strong effect in the lipophilic radicals generating system (Fig. 2). In the heterogeneous emulsion system, which was assumed to be a simple model of biological polyphasic systems, the effect of the antioxidant is thought to relate to the species of radicals and the polarity of the antioxidant. HER had hydrophilic antioxidants and contained $10 \%$ rosmarinic acid, which is a typical hydrophilic antioxidant. These ingredients would result in HER acting effectively against

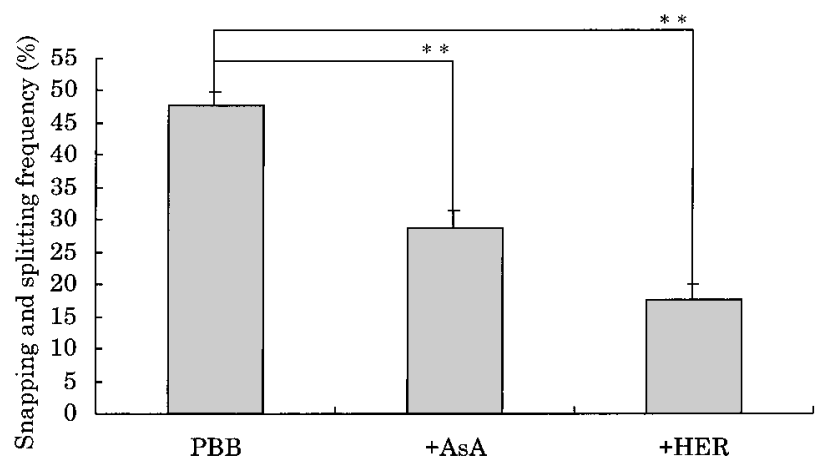

Fig. 7 Effects of HER on Snapping and Splitting Ends. Seven days after chemical treatment and antioxidant sample $(0.25 \%$ concentration $)$ application, the hair tress was brushed 5000 times. After brushing, snapping and splitting end of the hair was observed. Abbreviations are the same as in Fig. 4 and 5. Data was taken from the $\mathrm{M} \pm \mathrm{SD}$ of 3 samples. ** represents significantly different $(p<0.01)$ to PBB. 
hydrophilic radicals in a heterogeneous emulsion system.

Natural hairs have scale type layers, called cuticles, and they are covered on the surface by fatty acid layers. Previous papers reported that these fatty acid layers were exfoliated after chemical or physical treatments and the surface of the hair would be hydrophilic (25, 26). In hydrophilic conditions, HER effectively served as an antioxidant and could lead to preventing an increase in cysteic acid (Table 1), suppressing CL enhancement (Fig. 4-6) and also preventing an increase in the frequency of snapping and splitting (Fig. 7) in the damaged hair.

In the previous study (1), we demonstrated that chemical and physical stress, as well as environmental stress in daily life, could damage hair. We also showed that cysteic acid, which is an oxidative product, increased in the hair depending on the amount of stress in daily life. As shown in Table 1, HER application immediately after chemical treatment suppressed the increase in cysteic acid but application $7 \mathrm{~d}$ after treatment did not. It was thought that some environmental factors in the room atmosphere could lead to the oxidation in chemically treated hair, and HER could prevent the reaction in damaged hair.

CL measurement is a useful method for analyzing the oxidative condition in biological tissue $(15,16)$. In previous papers, it was reported that plant extracts from tropical regions were effective in skin lipid peroxidation (27), and these effects were confirmed with a CL analysis $(17,28)$. Using CL analysis methods, we also demonstrated that HER, which was hydrophilic extract, had an antioxidant effect on hair-damage that was subjected to usual hair treatment in daily life. In CL measurement using MCLA, both HER and AsA suppressed the CL level in the damaged hair (Fig. 4, 5), though only HER exhibited the antioxidant effect in the CL analysis without chemical reagents (Fig. 6). Furthermore, the effect of HER tended to be stronger than that of AsA concerning the prevention of snapping and splitting of hair. Using chemical reagents, such as MCLA, could enhance the CL level and lead to easier detection of CL. However, using the reagents may affect the results because antioxidants may react with the reagents and quench the CL. It was considered that using the system without the CL enhancing reagent, when directly evaluating the effect of antioxidants in the biological tissue, would reflect the oxidative state in biological tissue.

Benard et al. reported the measurement of CL in damaged hair, and they analyzed the CL immediately after UV irradiation (29). As shown in Fig. 1, the apparent CL peak was observed immediately after UV exposure. However it was concluded that this peak was derived from singlet oxygen in the hair because the peak was also observed without any sample. This CL peak also had the specific spectra of singlet oxygen (data not shown). In this study, we detected CL in hair that was subjected to short bursts of UV, and the CL analysis was started $5 \mathrm{~s}$ after exposure of UV. This neglected influence of singlet oxygen and we could evaluate the effect of HER in detail using this system.

In our previous study (1), we confirmed that physical hair stress in daily life, such as brushing and UV exposure, were also influences on hair-damage as well as is chemical treatment (e.g. perming, bleaching). We revealed the effect of HER on hair stress in this study. Hair dyeing is one of the most useful treatments to keep attractive hair. However, hydrogen peroxide $\left(\mathrm{H}_{2} \mathrm{O}_{2}\right)$ is most frequently used as an oxidizing agent for polymerized dye precursors in commercial hair dyeing compositions and it causes serious damages to hair. Recently we confirmed that polyphenol oxidase (laccase) could catalyze the oxidative reaction of the dye precursors and also dye the hair effectively without generating $\mathrm{H}_{2} \mathrm{O}_{2}$. We will report separately the details on the enzymatic reaction in hair dyeing (30).

\section{Acknowledgement}

We are grateful to Dr. Jyunichi Kimura for his kind direction of CL measurement.

\section{References}

1. K. TAKADA, A. NAKAMURA, N. MATSUO, A. INOUE, K. SOMEYA and H. SHIMOGAKI, Influence of Oxidative and/or Reductive Treatment on Human Hair (I): Analysis of Hair-Damage after Oxidative and/or Reductive Treatment, J. Oleo Sci., Vol. 52, 541-548 (2003).

2. W.A. PRYOR, The Formation of Free Radical and the Consequences of their Reactions in vivo, Photochem. Photobiol., Vol. 28, 787-801 (1978).

3. K. YAGI, Lipid Peroxides and Human Diseases, Chem. Phys. Lipids, Vol. 45, 337-351 (1987).

4. P.A. CERUTTI, Prooxidant States and Tumor Promotion, Science, Vol. 227, 337-351 (1985). 


\section{K. Takada, K. Someya, N. Matsuo et al.}

5. H.H. PRAPER and R.P. BIRD, Antioxidants and Cancer, J. Agric. Food Chem., Vol. 32, 433-435 (1984).

6. B.E. LEIBOVITEZ and B.V. SIEGEL, Aspect of Free Radical Reaction in Biological Systems: Aging, J. Gerontol., Vol. 35, 45-56 (1980).

7. D. HARMAN, M.L. HEIDRIC and D.E. EDDY, Free Radical Theory of Aging: Effect of Free-radical-reaction Inhibitories on Immune Response, J. Am. Geriatric. Soc., Vol. 25, 400-407 (1977).

8. T. SUZUKI, T. MIYAZAWA, K. FUJIMOTO, M. OHTSUKA and M. TSUTSUMI, Age-related Accumulation of Phosphatidylcholine Hydroperoxide in Cultured Human Diploid Cells and its Prevention by $\alpha$-Tocopherol, Lipids, Vol. 28, 775778 (1993).

9. T. MIYAZAWA, T. SUZUKI and K. FUJIMOTO, Age-dependent Accumulation of Phosphatidylcholine Hydroperoxide in the Brain and Liver of the Rat, Lipids, Vol. 28, 789-793 (1993).

10. L.S. YOSHIDA, T. MIYAZAWA, I. HATAYAMA, K. SATO, K. FUJIMOTO and T. KANEDA, Phosphatidylcholine Peroxidation and Liver Cancer in Mice Fed a Choline-deficient Diet with Ethionine, Free Radical Biol. Med., Vol. 14, 191-199 (1993).

11. K. SOMEYA, The Effect of Extracts from Rosmarinus officinalis L. on Food Preservation, Food Chemical, Vol. 1998-9, 46-50 (1998).

12. N. NAKATANI, Chemistry of Antioxidants from Labiatae Herbs, in Food Phytochemicals for Cancer Prevention II, (C-T. HO and T. OSAWA, eds.), American Chemical Society, Washington, pp. 144-153 (1994).

13. S. CHANG, Natural Antioxidants from Rosemary and Sage, $J$. Food Sci., Vol. 42, 1102-1106 (1977).

14. M.E. CUVELIER, Antioxidative Activity and Phenolic Composition of Pilot-plant and Commercial Extracts of Sage and Rosemary, J. Am. Oil Chem. Soc., Vol. 73, 645-652 (1996).

15. T. MIYAZAWA, T. SUZUKI, K. FUJIMOTO and K. YASUDA, Chemiluminescent Simultaneous Determination of Phosphatidylcoline Hydroperoxide and Phosphatidylethanolamine Hydroperoxide in the Liver and Brain of the Rat, J. Lipid Res., Vol. 33, 1051-1059 (1992).

16. T. MIYAZAWA, K. YASUDA and K. FUJIMOTO, Chemiluminescence-high Performance Liquid Chromatography of Phosphatidylcholine Hydroperoxide, Anal. Lett., Vol. 20, 915-925 (1987).

17. K. SOMEYA, H. SHIMIZU, C. UCHIYAMA, I. NAKAJIMA, T. HAYASHI, K. TAKADA, M. KUROYANAGI and T. MIYAZAWA, Antioxidant Effects of Caesalpinia paraensis Extract on Human Skin Lipid Peroxidation, J. Oleo Sci., Vol. 52, 463-470 (2003)

18. M. KIGAWA, S. KATAOKA, Y. OCHIAI and K. KANEKO,
Effects of APM on PMN Phagocytosis and ROS-induced Inflammation, J. Dent. Res., Vol. 80, 563 (2001).

19. W. PORTER, E.D. BLACK and A.M. DROLET, Use of Polyamide Oxidative Fluorescence Test on Lipid Emulsions: Contrast in Relative Effectiveness of Antioxidants in Bulk versus Dispersed Systems, J. Agric. Food Chem., Vol. 37, 615-624 (1989).

20. E. BRUNA, E. PETIT, M. BELJEAN-LEYMARIE, S. HUYNH and A. NOUVELOT, Specific Susceptibility of Docosahexaenoic Acid and Eicosapentaenoic Acid to Peroxidation in Aqueous Solution, Lipids, Vol. 24, 970-975 (1989).

21. S. HARA, T. SONE and Y. TOTANI, Antioxidative Activity of Nitrogen-containing Phospholipids toward Emulsified Fish Oils, J. Jpn Oil Chem. Soc., Vol. 49, 937-943 (2000).

22. V. BONDET, M.E. CUVELIER and C. BERSET, Behavior of Phenolic Antioxidants in a Partitioned Medium: Focus on Linoleic Acid Peroxidation Induced by Iron/Ascorbic Acid System, J. Am. Oil Chem. Soc., Vol. 77, 813-818 (2000).

23. M.E. CUVELIER, V. BONDET and C. BERSET, Behavior of Phenolic Antioxidants in a Partitioned Medium: Structure-activity Relationship, J. Am. Oil Chem. Soc., Vol. 77, 819-823 (2000).

24. E.N. FRANKEL, S.W. HUANG, J. KANNER and J.B. GERMAN, Interfacial Phenomena in the Evaluation of Antioxidants: Bulk Oils vs Emulsions, J. Agric. Food Chem., Vol. 42, 10541059 (1994).

25. J.A. SWIFT, Human Hair Cuticle: Biologically Conspired to the Owner's Advantage, J. Cosmet. Sci., Vol. 50, 23-47 (1999).

26. L.N. JONES and D.E. RIVETT, The Role of 18-Methyleicosanoic Acid in the Structure and Formation of Mammalian Hair Fibres, Micron, Vol. 28, 469-485 (1997).

27. K. SOMEYA, Y. TOTSUKA, M. MURAKOSHI, H. KITANO and T. MIYAZAWA, The Effect of Natural Carotenoid (Palm Fruit Carotene) Intake on Skin Lipid Peroxidation in Hairless Mice, J. Nutr. Sci. Vitaminol., Vol. 40, 303-314 (1994).

28. K. SOMEYA, Y. TOTSUKA, M. MURAKOSHI, H. KITANO and T. MIYAZAWA, The Antioxidant Effect of Palm Fruit Carotene on Skin Lipid Peroxidation in Guinea Pigs as Estimated by Chemiluminescence-HPLC Method, J. Nutr. Sci. Vitaminol., Vol. 40, 315-324 (1994).

29. S. BENARD, H. NERENZ, M. ROHR and K. SCHRADER, ICL-H (Induced Chemiluminescence of Human Hair) a New Method for Quantitative Analysis of Hair-stress, IFSCC Magazine, Vol. 4, 185-189 (2001).

30. K. TAKADA, T. UOZUMI, A. KIMURA, K. SOMEYA and T. YOSHINO, Influence of Oxidative and/or Reductive Treatment on Human Hair (III): Oxidative Reaction of Polyphenol Oxidase (Laccase) to Hair Dyeing, J. Oleo Sci., Vol. 52, 557-563 (2003). 